\title{
Inertial-range dynamics and scaling laws of two-dimensional magnetohydrodynamic turbulence in the weak field regime
}

\author{
Luke A. K. Blackbourn and Chuong V. Tran* \\ School of Mathematics and Statistics, University of St Andrews, \\ St Andrews, KY16 9SS, United Kingdom
}

(Dated: July 19, 2014)

\begin{abstract}
We study inertial-range dynamics and scaling laws in unforced two-dimensional magnetohydrodynamic turbulence in the regime of moderately small and small initial magnetic-to-kinetic energy ratio $r_{0}$, with an emphasis on the latter. The regime of small $r_{0}$ corresponds to a relatively weak field and strong magnetic stretching, whereby the turbulence is characterized by an intense conversion of kinetic into magnetic energy (dynamo action in the three-dimensional context). This conversion is an inertial-range phenomenon and, upon becoming quasi-saturated, deposits the converted energy within the inertial range rather than transferring it to the small scales. As a result, the magnetic energy spectrum $E_{\boldsymbol{b}}(k)$ in the inertial range can become quite shallow and may not be adequately explained or understood in terms of conventional cascade theories. It is demonstrated by numerical simulations at high Reynolds numbers (and unity magnetic Prandtl number) that the energetics and inertial-range scaling depend strongly on $r_{0}$. In particular, for fully developed turbulence with $r_{0}$ in the range [1/4,1/4096], $E_{\boldsymbol{b}}(k)$ is found to scale as $k^{\alpha}$, where $\alpha \gtrsim-1$, including $\alpha>0$. The extent of such a shallow spectrum is limited, becoming broader as $r_{0}$ is decreased. The slope $\alpha$ increases as $r_{0}$ is decreased, appearing to tend to +1 in the limit of small $r_{0}$. This implies equipartition of magnetic energy among the Fourier modes of the inertial range and the scaling $k^{-1}$ of the magnetic potential variance, whose flux is direct rather than inverse. This behavior of the potential resembles that of a passive scalar. However, unlike a passive scalar whose variance dissipation rate slowly vanishes in the diffusionless limit, the dissipation rate of the magnetic potential variance scales linearly with the diffusivity in that limit. Meanwhile, the kinetic energy spectrum is relatively steep, followed by a much shallower tail due to strong antidynamo excitation. This gives rise to a total energy spectrum poorly obeying a power-law scaling.
\end{abstract}

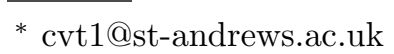




\section{INTRODUCTION}

Magnetohydrodynamic turbulence differs from its usual counterpart in some fundamental aspects. In the former, dynamo action can convert a substantial amount of kinetic energy into magnetic energy. This phenomenon, which has become a subject of active research [1-11], gives rise to several interesting effects. First, dynamo action may help to rid the large scales of kinetic energy, an addition to the usual loss of kinetic energy due to the direct energy transfer through the vortex stretching mechanism. Second, dynamo action, upon becoming saturated (or quasi-saturated), may deposit a substantial amount of the converted magnetic energy in the inertial range. Hence, the magnetic modes in this range may be more strongly excited than those of a passively advected field. For strong dynamo action, the inertial range may no longer be considered an energy-free "conduit", through which the energy is transferred to the dissipation scales. This is in a sharp contrast to fluid systems governed by the three-dimensional Navier-Stokes, surface quasi-geostrophic and Burgers equations, whose inertial ranges are virtually devoid of energy. Third, upon losing energy to dynamo action, the velocity field becomes weaker, necessarily rendering less effective magnetic and vortex stretching. Arguably, the delineation of these effects is essential for a detailed understanding of the turbulence. Nonetheless, relatively successful theories in the literature have neither directly addressed nor been concerned with these apparent effects [12-15]. Note, however, that Iroshnikov [12] and Kraichnan [13] envisaged a reduction of energy transfer by Alfvén waves. This is in a qualitative agreement with the weakening of magnetic and vortex stretching by dynamo action presently discussed.

In two dimensions, energy conversion plays a particularly prominent role in the inertialrange dynamics and energy transfer. The reason is that in the absence of genuine vortex stretching, the Lorentz force is solely responsible for vortex amplification. This amplification has been known to give rise to bounded enstrophy, even in the limit of zero magnetic Prandtl number [16]. As a result, the dissipation of kinetic energy vanishes in this limit and the conversion of kinetic into magnetic energy becomes the only mechanism through which the large scales can rid themselves of kinetic energy. However, since this conversion exhausts rather than excites the mechanical modes, the reverse process (referred to in Blackbourn and Tran [17] and this study as antidynamo action) is required to excite and replenish the mechanical modes (particularly those in the inertial range) if a persistent energy flux is to 
be realizable. Hence, antidynamo action also plays a key role in the energy transfer. A detailed analysis of the problem of energy conversion responsible for the energy transfer can be found in a recent study by Blackbourn and Tran [17]. A brief review of this problem is given in $\S$ II for convenience.

An important parameter in two-dimensional magnetohydrodynamic turbulence is the magnetic-to-kinetic energy ratio, say $r(t)$. Apparently, the regime $r \ll 1$ corresponds to a relatively weak field and strong magnetic stretching, whereby the dynamics should be dominated by an intense conversion of kinetic into magnetic energy. On the other hand, the regime $r \gg 1$ corresponds to relatively weak advection (weak magnetic stretching), whereby a conversion of magnetic into kinetic energy could be a key dynamical feature. We are not aware of comprehensive investigations of these extreme regimes. Recently, Blackbourn and Tran [17] studied the energetics and inertial-range scaling laws of two-dimensional magnetohydrodynamic turbulence at unity magnetic Prandtl number $(P m=1)$ for moderate values of $r_{0}=r(0)$ in the range $[1 / 16,16]$. It was found that for fully developed turbulence, the energy conversion, upon becoming saturated (dynamo saturation in the three-dimensional context), strongly excites the inertial range as expected. The kinetic, magnetic and total energy spectra in this range appear to depend on $r_{0}$. Most notably, the total energy spectrum was observed to be significantly shallower than $k^{-3 / 2}$, particularly for the cases with lower $r_{0}$. These dynamical features do not appear to be adequately addressed by available theories $[12-15,18]$. Hence further investigation is highly desirable.

This study extends the results of Blackbourn and Tran [17] with an aim to better understand the dynamical features described in the preceding paragraph, by doubling the resolution from $4096 \times 4096$ to $8192 \times 8192$ and broadening the range of $r_{0}$ toward the weak field regime, down to $r_{0}=1 / 4096$. This allows us to examine in detail the dependence of the inertial-range dynamics and scaling laws on $r_{0}$, for $r_{0} \ll 1$. Another aim is to address in a quantitative manner the observation by Blackbourn and Tran [17] that strong conversion of kinetic into magnetic energy can suppress the inverse transfer of the magnetic potential variance. It is demonstrated by numerical simulations at $P m=1$ and for $r_{0} \in[1 / 4,1 / 4096]$ that the magnetic energy spectrum is much shallower than its kinetic counterpart. The sum of these highly "incongruous" spectra, i.e. the total energy spectrum, poorly approximates a power-law scaling. The slope of the magnetic energy spectrum is found to increase dramatically with $r_{0}$, appearing to tend to +1 in the small $r_{0}$ limit. This scaling corresponds 
to equipartition of magnetic energy among individual Fourier modes and equipartition of magnetic potential variance among the wave-number octaves in the inertial range. The transfer of the magnetic potential variance, induced by strong energy conversion, is direct rather than inverse. This is reminiscent of the dynamics of a passive scalar advected by a large-scale flow. Note, however, that there are fundamental differences between the passive scalar and the present cases. In the former, the mean-square scalar gradient can be amplified without bound in the diffusionless and infinite-time limits, while in the latter, the magnetic energy is bounded uniformly in time for bounded initial total energy. In some sense, the direct transfer of a passive scalar can be considered relatively more spontaneous than that of the magnetic potential. Another difference is that antidynamo interaction is an integral part of the magnetic potential dynamics. Hence the magnetic potential is an active scalar in its own right.

\section{THEORETICAL BACKGROUND}

The evolution of the magnetic induction and the motion of a two-dimensional incompressible and electrically conductive fluid are governed by

$$
\begin{aligned}
& \frac{\partial \omega}{\partial t}+J(\psi, \omega)=J(a, \Delta a)+\nu \Delta \omega \\
& \frac{\partial a}{\partial t}+J(\psi, a)=\mu \Delta a
\end{aligned}
$$

Here $\psi$ is the stream function, $\omega=\Delta \psi$ is the vorticity, $a$ is the magnetic potential, $J(\cdot, \cdot)$ denotes the Jacobian and $\nu$ and $\mu$ are the kinematic viscosity and magnetic diffusivity, respectively. The divergence-free fluid velocity $\boldsymbol{u}$ and magnetic induction $\boldsymbol{b}$ are given in terms of $\psi$ and $a$ by $\boldsymbol{u}=\left(-\psi_{y}, \psi_{x}\right)$ and $\boldsymbol{b}=\left(a_{y},-a_{x}\right)$, where subscripts denote differentiation.

The current density $j$ is given by $j=-\Delta a$. For convenience, we consider a doubly periodic domain, over which all the fields involved have zero average.

Let $\langle\cdot\rangle$ denote the domain average. The evolution (conversion and decay) of the kinetic energy $\left\langle|\nabla \psi|^{2}\right\rangle / 2=E_{\boldsymbol{u}}$ and magnetic energy $\left\langle|\nabla a|^{2}\right\rangle / 2=E_{\boldsymbol{b}}$ (per unit area) are governed by

$$
\begin{aligned}
& \frac{1}{2} \frac{d}{d t}\left\langle|\nabla \psi|^{2}\right\rangle=\langle\psi J(a, j)\rangle-\nu\left\langle\omega^{2}\right\rangle=\langle\psi J(a, j)\rangle-\epsilon_{\boldsymbol{u}}, \\
& \frac{1}{2} \frac{d}{d t}\left\langle|\nabla a|^{2}\right\rangle=-\langle j J(\psi, a)\rangle-\mu\left\langle j^{2}\right\rangle=-\langle j J(\psi, a)\rangle-\epsilon_{\boldsymbol{b}} .
\end{aligned}
$$


When $\nu=\mu=0$, the total energy $E=E_{\boldsymbol{u}}+E_{\boldsymbol{b}}$ is conserved as the triple-product terms on the right-hand sides of (3) and (4) cancel each other. These terms represent the conversion between $E_{\boldsymbol{u}}$ and $E_{\boldsymbol{b}}$ and play a key role in the transfer of $E$ to small scales (direct transfer). Note that the variance of the magnetic potential $\left\langle a^{2}\right\rangle$ (among other quantities) is conserved. The conservation of $\left\langle a^{2}\right\rangle$ prohibits significant kinetic-to-magnetic energy conversion from taking place at large scales [17]. Further implications of this conservation law are discussed in $\S$ III. On phenomenological grounds, $\left\langle a^{2}\right\rangle$ is expected to be transferred to large scales [1921]. The inverse transfer of $\left\langle a^{2}\right\rangle$, however, is relatively weak due to amplification of $E_{\boldsymbol{b}}$ [17]. In $\S$ III, this weak transfer can be seen to reverse direction by strong kinetic-to-magnetic energy conversion.

The respective kinetic, magnetic, and total energy dissipation rates $\epsilon_{\boldsymbol{u}}(t), \epsilon_{\boldsymbol{b}}(t)$ and $\epsilon(t)=$ $\epsilon_{\boldsymbol{u}}(t)+\epsilon_{\boldsymbol{b}}(t)$ are key dynamical parameters. Recent mathematical studies [16, 22, 23] have derived important constraints on these quantities. In particular, given $\mu>0,\left\langle\omega^{2}\right\rangle$ and $\left\langle j^{2}\right\rangle$ were shown to be uniformly bounded in time independently of $\nu$. It follows immediately that in the regime of small magnetic Prandtl number Pm, $\epsilon_{\boldsymbol{u}}$ decreases linearly with Pm. This may have far reaching implications, which have yet to be fully explored. In $\S$ III, the dependence of $\epsilon_{\boldsymbol{u}}(t), \epsilon_{\boldsymbol{b}}(t)$ and $\epsilon(t)$ on $r_{0}$ and on $\nu$ at $P m=1$ is examined.

In wave-number space, the exact cancellation of the triple-product terms on the righthand sides of (3) and (4), i.e. the conservation of total energy, manifests itself through interacting wave triads, each conserving energy individually. To see this, consider a vector triad $\boldsymbol{k}=\boldsymbol{\ell}+\boldsymbol{m}$ with the associated modal energy components $k^{2}\left|\widehat{\psi}_{\boldsymbol{k}}\right|^{2} / 2, \ell^{2}\left|\widehat{a}_{\boldsymbol{\ell}}\right|^{2} / 2$ and $m^{2}\left|\widehat{a}_{\boldsymbol{m}}\right|^{2} / 2$. Here $k=|\boldsymbol{k}|, \ell=|\ell|, m=|\boldsymbol{m}|$ and $\widehat{\psi}_{\boldsymbol{k}}$ and $\widehat{a}_{\boldsymbol{k}}$ denote the Fourier transforms of $\psi$ and $a$, respectively. The equations governing the energy transfer (without diffusion) within this triad are given by [17]

$$
\begin{aligned}
\frac{d}{d t} k^{2}\left|\widehat{\psi}_{\boldsymbol{k}}\right|^{2} & =\left(\ell^{2}-m^{2}\right) \boldsymbol{\ell} \times \boldsymbol{m}\left(\widehat{\psi}_{\boldsymbol{k}}^{*} \widehat{a}_{\ell} \widehat{a}_{\boldsymbol{m}}+\widehat{\psi}_{\boldsymbol{k}} \widehat{a}_{\ell}^{*} \widehat{a}_{\boldsymbol{m}}^{*}\right), \\
\frac{d}{d t} \ell^{2}\left|\widehat{a}_{\ell}\right|^{2} & =-\ell^{2} \boldsymbol{\ell} \times \boldsymbol{m}\left(\widehat{\psi}_{\boldsymbol{k}}^{*} \widehat{a}_{\ell} \widehat{a}_{\boldsymbol{m}}+\widehat{\psi}_{\boldsymbol{k}} \widehat{a}_{\ell}^{*} \widehat{a}_{\boldsymbol{m}}^{*}\right), \\
\frac{d}{d t} m^{2}\left|\widehat{a}_{\boldsymbol{m}}\right|^{2} & =m^{2} \boldsymbol{\ell} \times \boldsymbol{m}\left(\widehat{\psi}_{\boldsymbol{k}}^{*} \widehat{a}_{\ell} \widehat{a}_{\boldsymbol{m}}+\widehat{\psi}_{\boldsymbol{k}} \widehat{a}_{\ell}^{*} \widehat{a}_{\boldsymbol{m}}^{*}\right),
\end{aligned}
$$

where $\boldsymbol{\ell} \times \boldsymbol{m}=\ell_{x} m_{y}-\ell_{y} m_{x}$ and the asterisk denotes a complex conjugate. The terms on the right-hand sides of (5), (6) and (7) sum up to zero, indeed implying energy conservation within individual triads. Note that the magnetic potential variance is also conserved by individual triads as is clear from (6) and (7). 
A simple analysis of (5), (6) and (7) in Blackbourn and Tran [17] concludes that energy conversion and transfer go hand in hand and are facilitated by two distinct types of triads. One of these converts kinetic to magnetic energy, i.e. negative growth of $k^{2}\left|\widehat{\psi}_{\boldsymbol{k}}\right|^{2}$ in Eq. (5), while the other converts magnetic to kinetic energy, i.e. positive growth of $k^{2}\left|\widehat{\psi}_{\boldsymbol{k}}\right|^{2}$ in Eq. (5). For convenience, these triads are referred to as dynamo and antidynamo triads, respectively. For a qualitative behavior of these triads, see Fig. 1. The former helps to rid the large scales of kinetic energy and gives rise to a direct magnetic energy flux. Hence, the operation of dynamo triads is crucial in the energy transfer. However, since dynamo triads themselves exhaust rather than excite the mechanical modes, their persistent operation in the inertial range is impossible without help from antidynamo triads to excite and replenish these modes. As far as the direct energy transfer is concerned, this help comes with a cost as antidynamo interaction involves an inverse magnetic energy flux (see Fig. 1). It is not known with precision to what extent this flux can neutralize its direct counterpart. Nonetheless, a nearly complete neutralization is possible in fully developed turbulence, where dynamo saturation in three dimensions and energy conversion saturation in two dimensions are widely observed $[3,11,17]$. Note that dynamo triads involving large-scale mechanical modes can be fully operative independently of antidynamo triads, provided that their mechanical modes have yet to be exhausted. These triads represent large-scale advection and require a magnetic energy spectrum not steeper than $k^{-1}$ for a nonvanishing magnetic energy flux [24]. This corresponds to a relatively weak transfer, in the sense that a highly excited inertial range is required. Given the cancellation (whether partial or nearly total) between the dynamo and antidynamo fluxes and the weak transfer by large-scale advection, the direct energy transfer in two-dimensional magnetohydrodynamic turbulence can be expected to be relatively weak. Indeed, Blackbourn and Tran [17] found energy spectra significantly shallower than the classical $k^{-5 / 3}$ spectrum (in fact, shallower than the Iroshnikov and Kraichnan $k^{-3 / 2}$ spectrum) and suggested the possibility of a $k^{-1}$ scaling. This scaling is found to be a poor approximation to the present numerical results for small $r_{0}$. Indeed, it can be seen in $\S$ III that the magnetic energy spectrum closely obeys the power-law scaling $k^{\alpha}$, where $\alpha$ becomes positive for small $r_{0}$. On the other hand, the kinetic energy spectrum is relatively steep, though with a shallow tail due to antidynamo excitation of the small scales. The sum of these highly "incongruous" spectra gives rise to a total energy spectrum poorly approximated by a power-law scaling. 

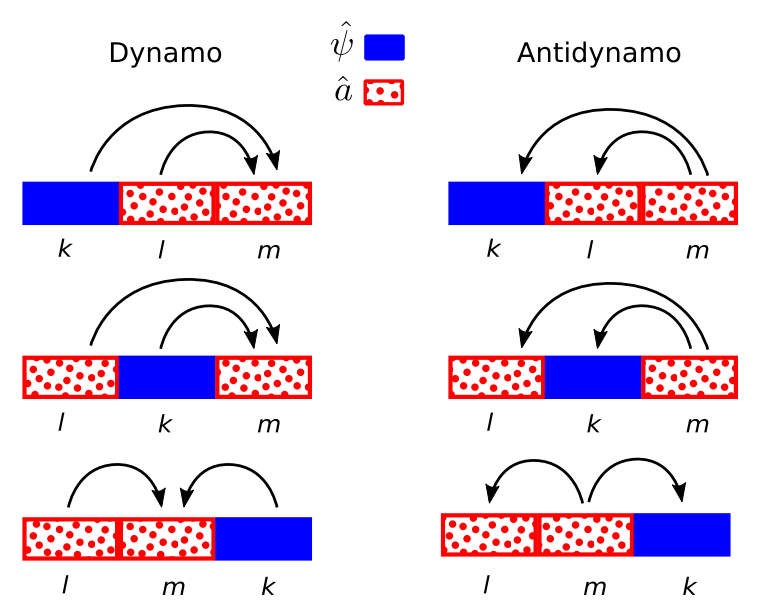

FIG. 1: (Color online) A schematic description of dynamo and antidynamo wave triads $\boldsymbol{k}=\boldsymbol{\ell}+\boldsymbol{m}(\ell=|\ell|<m=|\boldsymbol{m}|)$ responsible for energy conversion and transfer. The arrows indicate the direction of energy transfer.

\section{NUMERICAL RESULTS}

We now present and discuss our simulation results. Equations (1) and (2) were numerically integrated in a doubly periodic domain of side $2 \pi$ using a pseudo-spectral method. The time-stepping procedure was a fourth order Runge-Kutta method, with the dissipative terms incorporated exactly using integrating factors. Aliasing errors were removed at each timestep using a high-order spectral smoothing operator as described in [25]. In all simulations, the initial magnetic field was confined to the wave-number range $[4,6]$, and its Fourier modes within this range were given random phases and equal magnitudes. We used localized and fully developed velocity fields in separate sets of simulations. This allows us to understand the different effects of these fields on the magnetic field dynamics. In the first two sets of simulations the velocity field was initialized in the same fashion as its magnetic counterpart, albeit being confined to the wave-number range [5, 7]. Both initial fields were then rescaled to produce various $r_{0}$. In the third set this seed velocity field was first evolved according to the two-dimensional Navier-Stokes equations until the time of maximum enstrophy dissipation, when the turbulence could be considered fully developed. At this time it was noted that the enstrophy spectrum (not shown) was close to the classical $k^{-1}$ form for about a decade of wave numbers. This field was then used as the initial velocity field (see Fig. 2). While the energy is virtually unchanged during the initial nonmagnetic evolution, it should be noted that the enstrophy does decay by about $10 \%$. The first set of 

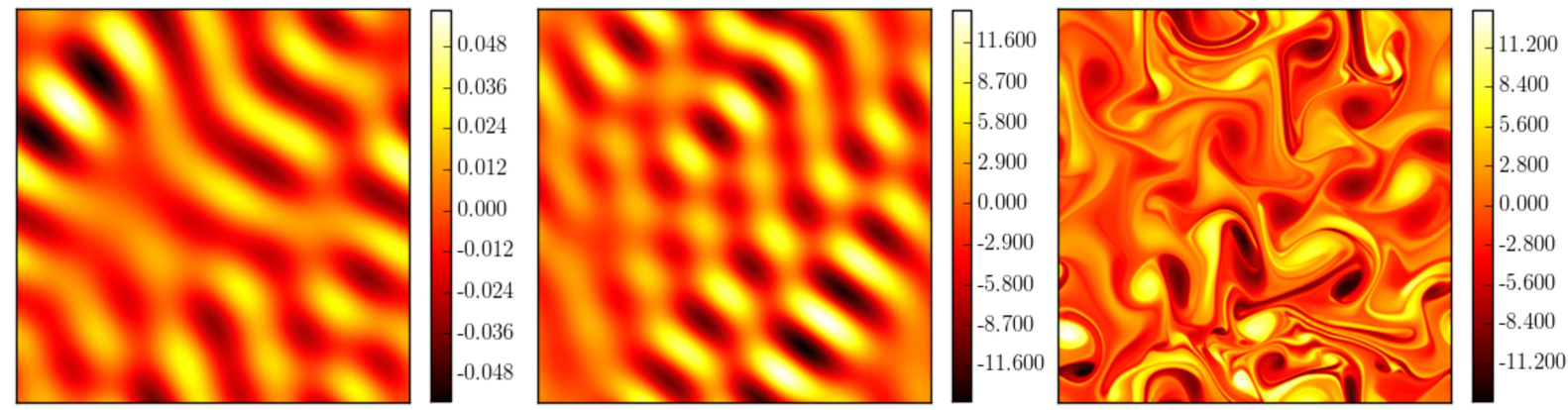

FIG. 2: (Color online) Representative initial magnetic potential field (left) and spectrally localized (center) and fully developed (right) initial vorticity fields used in the simulations, presented here for $r_{0}=1 / 256$.

simulations corresponds to $r_{0}=1 / 4,1 / 16,1 / 64,1 / 256,1 / 1024$, with unity total energy. For each $r_{0}$, four simulations at resolutions of $1024 \times 1024,2048 \times 2048,4096 \times 4096$ and $8192 \times 8192$ were run, with $\nu=\mu$ taking values $9.60 \times 10^{-4}, 3.81 \times 10^{-4}, 1.51 \times 10^{-4}$ and $6.00 \times 10^{-5}$ respectively. This represents a decrease in viscosity by a factor of $2^{4 / 3}$ when the resolution is doubled, as suggested by the estimates for the number of degrees of freedom derived by Tran and Yu [26]. The choice of $P m=1$ is mainly for computational expediency rather than physical relevance. Actual Prandtl numbers vary widely depending on the physical situation, with the solar tachocline and liquid metals typically having $P m$ in the range $\left[10^{-5}, 10^{-3}\right]$. The second and third set correspond to $r_{0}=1 / 256,1 / 1024,1 / 4096$, with $E_{\boldsymbol{b}}(0)=0.001$ fixed. This amounts to a total energy of 4.097 for the case $r_{0}=1 / 4096$. For each $r_{0}$, one simulation at $8192 \times 8192$ grid points was run, with $\nu=\mu=7.9 \times 10^{-5}$. Note that an increase in $\nu$ and $\mu$ in these simulations compared with the previous ones at the same resolution is necessary due to the more energetic initial condition for $r_{0}=1 / 4096$.

\section{A. Energy budget}

Fig. 3 shows the evolution of the energy components $E_{\boldsymbol{u}}(t)$ and $E_{\boldsymbol{b}}(t)$ and their ratio $r(t)=E_{\boldsymbol{b}} / E_{\boldsymbol{u}}$. For all simulations, strong magnetic stretching is a prominent dynamical feature during the early stage. For $r_{0}=1 / 4,1 / 16, r(t)$ becomes quasi-steady upon attaining values about 2.0. This is in agreement with the findings of Blackbourn and Tran [17], 
where the initial fields are twice as energetic, and of Biskamp and Welter [27]. From the highest resolution runs (least diffusive effects), it can be seen that (maximal) amplification of magnetic energy is approximately by threefold for $r_{0}=1 / 4$, eightfold for $r_{0}=1 / 16$, twentyfold for $r_{0}=1 / 64$, fortyfold for $r_{0}=1 / 256$ and fiftyfold for $r_{0}=1 / 1024$. The case $r_{0}=1 / 64$ appears critical, in the sense that magnetic stretching is barely able to give rise to a parity of $E_{\boldsymbol{u}}(t)$ and $E_{\boldsymbol{b}}(t)$ (at $t \approx 1.7$ ). Note that the conservation of $\left\langle a^{2}\right\rangle$ imposes a stiff constraint on magnetic energy amplification in the spectral vicinity of the magnetic reservoir, but in principle allowing for this amplification to proceed toward smaller scales until saturation. It is not known whether there exists a bound for the ratio $r_{\text {peak }} / r_{0}$, where $r_{\text {peak }}$ denotes the peak value of $r(t)$, as $r_{0} \rightarrow 0$. Further results below suggest a negative answer to this question. In any case, $r(t)$ diminishes in the limit $r_{0} \rightarrow 0$. Indeed, Fig. 3 indicates that for $r_{0}=1 / 64,1 / 256,1 / 1024$, the relation between $r_{\text {peak }}$ and $r_{0}$ is virtually linear.

The ratio $r_{\text {peak }} / r_{0}$ is a measure of the relative strength of magnetic stretching. As can be seen from the discussion above, this ratio increases as $r_{0}$ is decreased. The asymptotic behavior of $r_{\text {peak }} / r_{0}$ in the limit $r_{0} \rightarrow 0$ (and limit of ideal dynamics) is interesting. This problem is explored in some detail on the basis of the results from the second set of simulations.

Although the evolution of the total energy $E$ is not shown explicitly, one can deduce from Fig. 3 that $E$ decays most rapidly for the case $r_{0}=1 / 64$, where an approximate parity of $E_{\boldsymbol{u}}(t)$ and $E_{\boldsymbol{b}}(t)$ is attained for fully developed turbulence. Qualitatively speaking, this parity ensures both effective magnetic stretching and relatively strong antidynamo excitation of the mechanical modes in the inertial range, required for optimal dissipation. Toward the small $r_{0}$ regime, $E$ becomes better conserved as expected. Also for $r_{0}=1 / 64$ (and $\left.r_{0}=1 / 256\right), E_{\boldsymbol{u}}(t)$ becomes smaller (for $t>1$ ) as $\nu$ is decreased. This somewhat counter-intuitive behavior can be understood by noting the relatively strong surge in $E_{\boldsymbol{b}}(t)$ in response to weaker diffusion.

Fig. 4 shows the evolution of the energy dissipation rates $\epsilon_{\boldsymbol{u}}(t), \epsilon_{\boldsymbol{b}}(t)$ and $\epsilon(t)$. For similar but twice as energetic initial fields and for $r_{0}=1 / 4,1 / 16$, it was observed by Blackbourn and Tran [17] that each of these rates achieve a smaller maximum at increasingly greater time as $\nu$ is decreased. This is no longer the case for lower $r_{0}$. More precisely, when $r_{0}=1 / 256,1 / 1024$ (and sufficiently small $\nu$ ), each of these rates achieve a greater maximum at increasingly greater time as $\nu$ is decreased. This behavior is consistent with the possibility of positive limiting dissipation rate achievable in infinite time. 

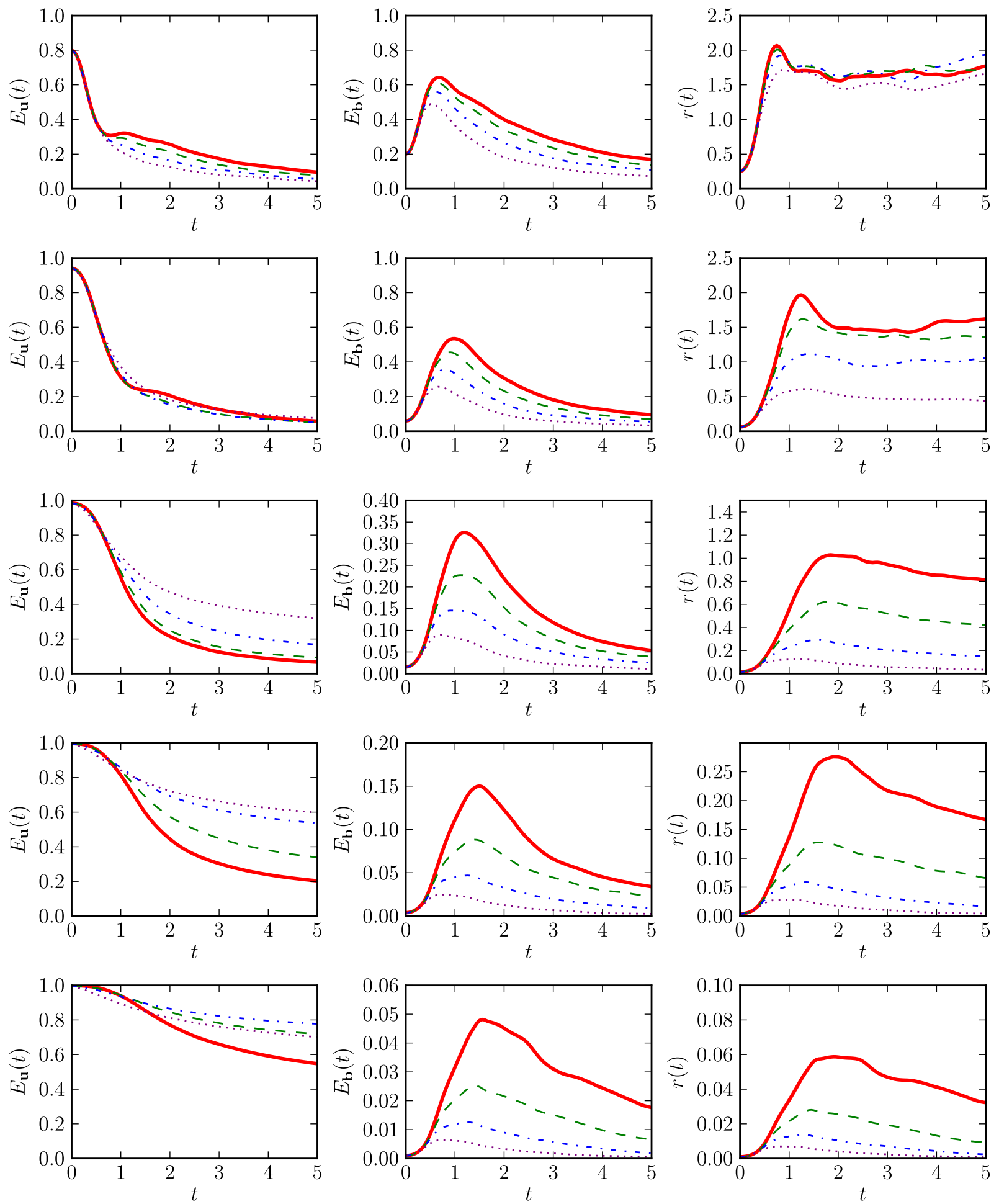

FIG. 3: (Color online) Kinetic energy $E_{\boldsymbol{u}}(t)$, magnetic energy $E_{\boldsymbol{b}}(t)$ and their ratio $r(t)=E_{\boldsymbol{b}} / E_{\boldsymbol{u}}$ versus $t$ for numerical simulations with resolutions $1024 \times 1024$ (dotted), $2048 \times 2048$ (dashed-dotted), $4096 \times 4096$ (dashed) and $8192 \times 8192$ (solid) and $r_{0}=1 / 4,1 / 16,1 / 64,1 / 256,1 / 1024$. The rows are in decreasing order of $r_{0}$. 

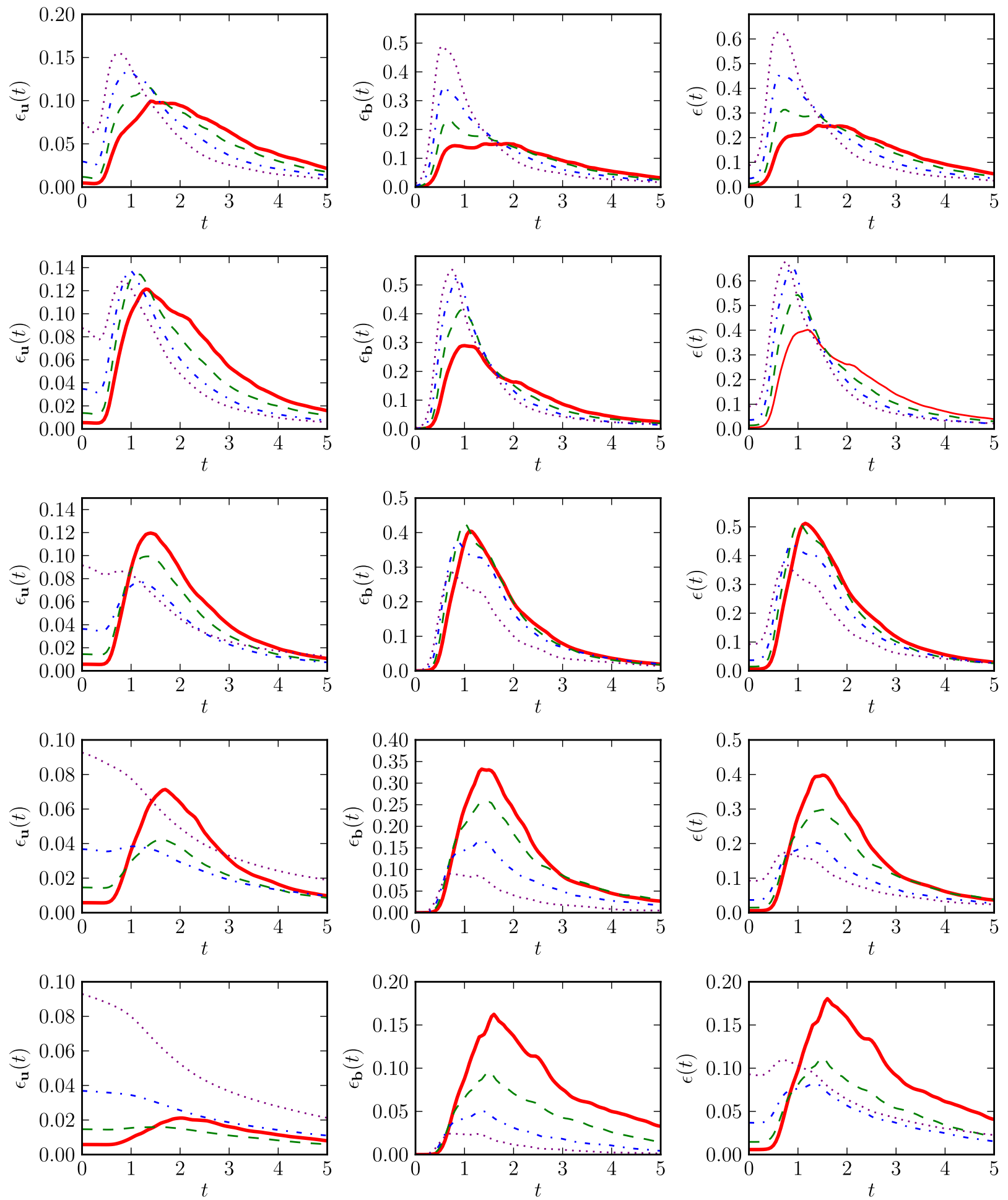

FIG. 4: (Color online) Kinetic, magnetic and total energy dissipation rates $\epsilon_{\boldsymbol{u}}(t), \epsilon_{\boldsymbol{b}}(t)$ and $\epsilon(t)$, respectively, versus $t$ for numerical simulations with resolutions $1024 \times 1024$ (dotted), $2048 \times 2048$ (dashed-dotted), $4096 \times 4096$ (dashed) and $8192 \times 8192$ (solid) and $r_{0}=1 / 4,1 / 16,1 / 64,1 / 256,1 / 1024$. The rows are in decreasing order of $r_{0}$. 

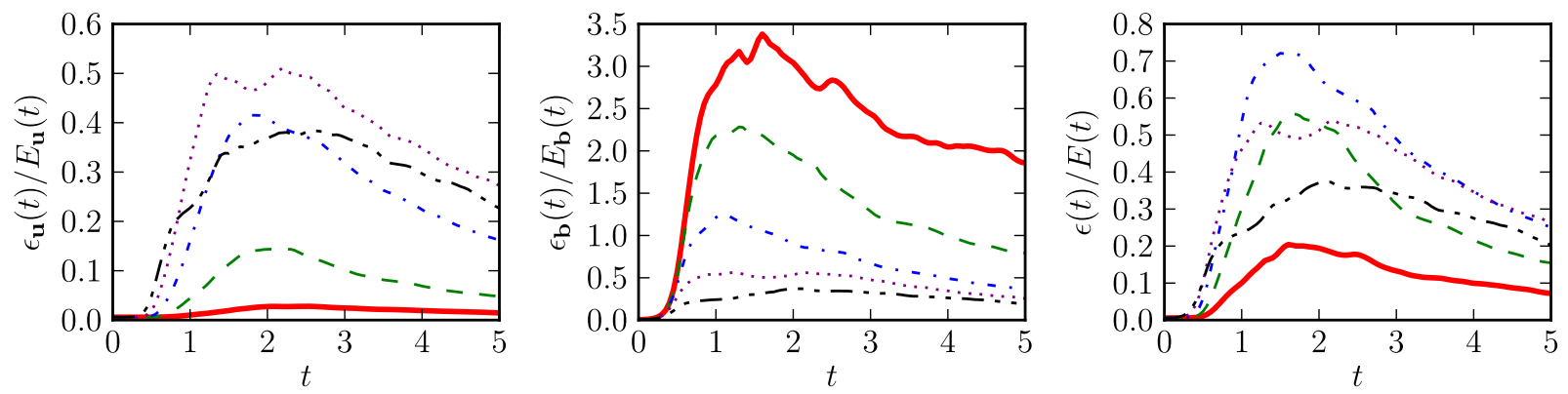

FIG. 5: (Color online) Evolution of the exponential energy dissipation rates $\epsilon_{\boldsymbol{u}} / E_{\boldsymbol{u}}, \epsilon_{\boldsymbol{b}} / E_{\boldsymbol{b}}$ and $\epsilon / E$ for the highest resolution runs, with $r_{0}=1 / 4,1 / 16,1 / 64,1 / 256$ and $1 / 1024$ given by the dashed-double-dotted, dotted, dashed-dotted, dashed and solid lines, respectively.

The exponential energy dissipation rates (energy dissipation rates per unit energy) $\epsilon_{\boldsymbol{u}} / E_{\boldsymbol{u}}$, $\epsilon_{\boldsymbol{b}} / E_{\boldsymbol{b}}$ and $\epsilon / E$ versus time for the highest resolution runs are plotted in Fig. 5. The case $r_{0}=1 / 64$ corresponds to optimal dissipation (highest peak in $\epsilon / E$ ) as observed earlier. The rapid decrease of $\epsilon_{\boldsymbol{u}} / E_{\boldsymbol{u}}$ toward the weak field regime is fully expected as kinetic energy is an inviscid invariant of Navier-Stokes turbulence. Given $r \ll 1$, this decrease entails a corresponding decrease in $\epsilon / E$. What is interesting is the increase of $\epsilon_{\boldsymbol{b}} / E_{\boldsymbol{b}}$ and its relatively large magnitude, implying an increasingly shallow magnetic energy spectrum in the inertial range.

Fig. 6 shows the respective kinetic, magnetic and total energy spectra $E_{\boldsymbol{u}}(k), E_{\boldsymbol{b}}(k)$ and $E(k)$ at time $t=2.5$, which is well after the time of peak energy dissipation, when the turbulence can be considered fully developed. Across the board, the inertial range becomes shallower for higher resolutions as expected. The kinetic energy reservoir becomes less depleted for lower $r_{0}$ as a smaller amount of kinetic energy per unit kinetic energy may be converted. In the energy inertial range of the lower- $r_{0}$ cases, $E_{\boldsymbol{u}}(k)$ is significantly steeper than $k^{-1}$, though with a relatively shallower tail due to antidynamo excitation. Meanwhile $E_{\boldsymbol{b}}(k)$ is surprisingly shallow (with limited extent), approximately $k^{1}$. Thanks to the latter, $E(k)$ is slightly shallower than $k^{-1}$. Note that a $k^{1}$ scaling corresponds to equipartition of magnetic energy among the Fourier modes of the inertial range. An interesting question is whether this behavior is universal for the weak field limit. Evidence for a positive answer to this question is presented shortly.

It is evident from the observed energy spectra, particularly from those for the relatively weak field cases, that energy tends to linger in the inertial range rather than to cascade 

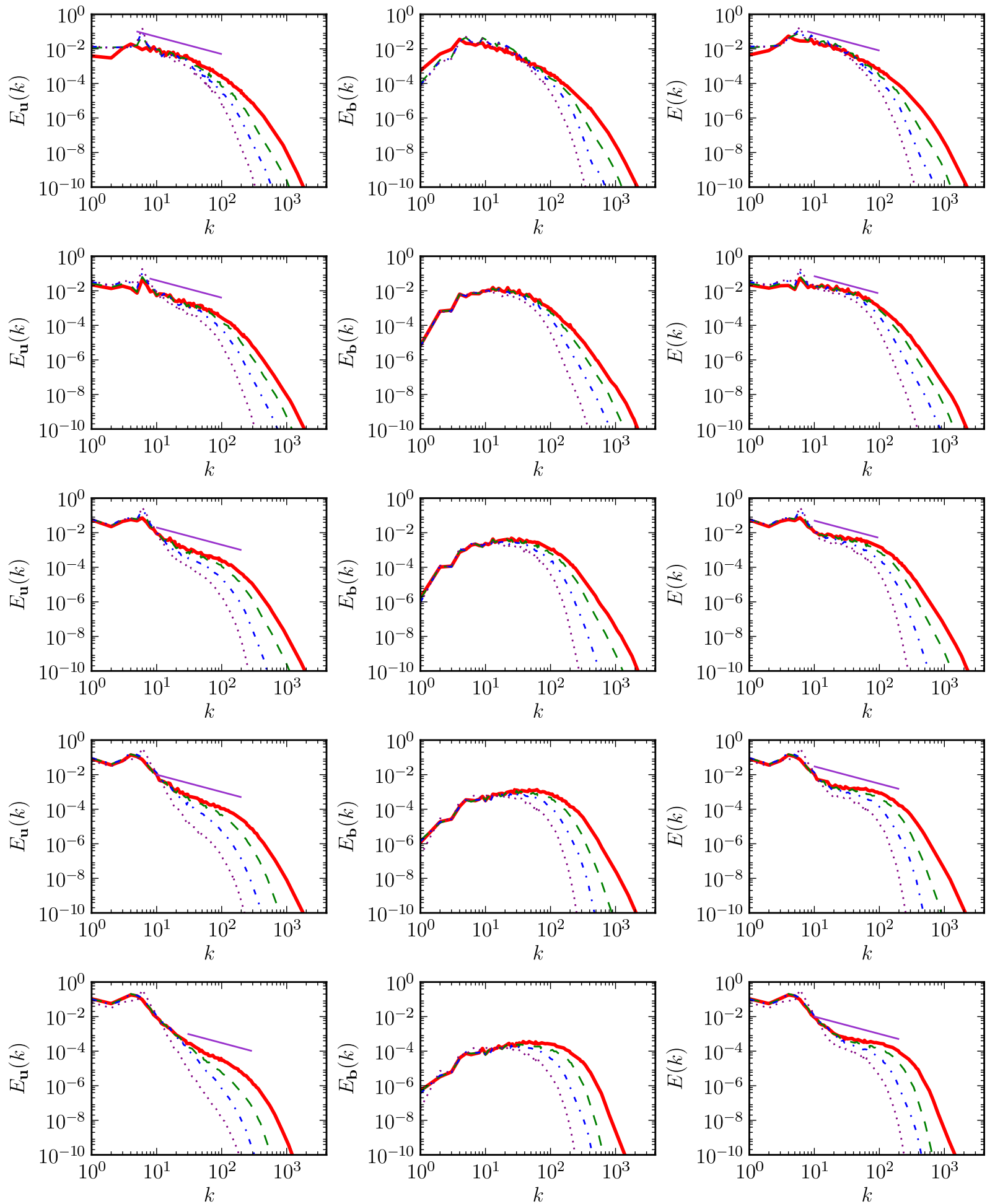

FIG. 6: (Color online) Kinetic, magnetic and total energy spectra $E_{\boldsymbol{u}}(k), E_{\boldsymbol{b}}(k)$ and $E(k)$, respectively, for fully developed turbulence (at $t=2.5$ ), for numerical simulations with resolutions $1024 \times 1024$ (dotted), $2048 \times 2048$ (dashed-dotted), $4096 \times 4096$ (dashed) and $8192 \times 8192$ (solid) and $r_{0}=1 / 4,1 / 16,1 / 64,1 / 256,1 / 1024$. The rows are in decreasing order of $r_{0}$. The referen₫马 lines have a slope of -1 . 

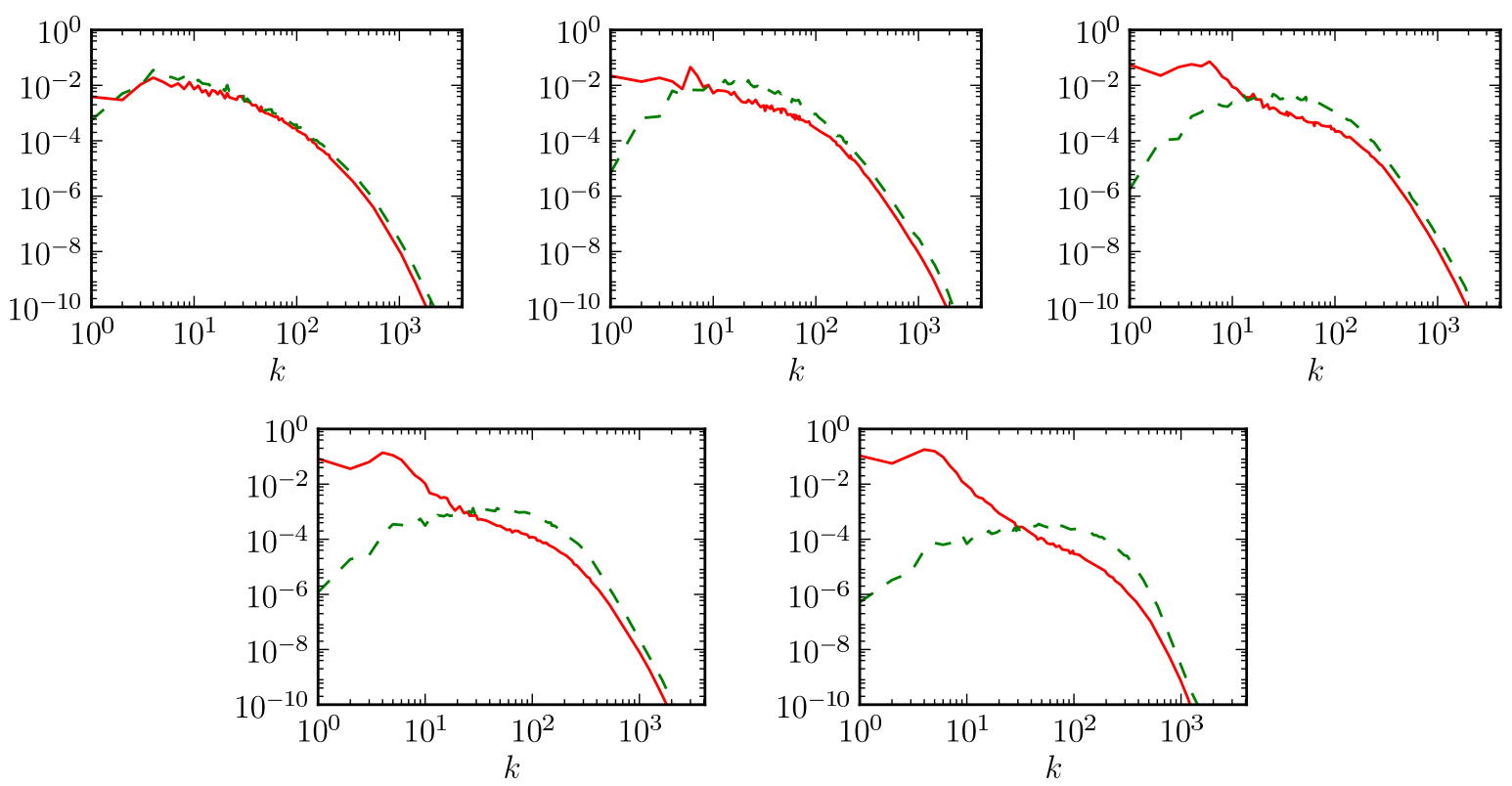

FIG. 7: (Color online) Kinetic (solid) and magnetic (dashed) energy spectra for the highest resolution from Fig. 6 in decreasing order of $r_{0}$ from top left to bottom right.

to the dissipation range. This is an indication of weak energy transfer discussed in the preceding sections.

The magnetic modes in the higher-wave-number end of the inertial range and in the dissipation range are more strongly excited than their neighboring mechanical modes (see Fig. 7). This discrepancy in the level of excitation becomes more conspicuous for lower $r_{0}$. As discussed in the preceding section, the said mechanical modes are excited and replenished by their magnetic counterparts through antidynamo interaction. On physical grounds, it is plausible to expect the "excitees" to remain weaker than their "excitors". Our results are consistent with this expectation. The implication is that energy equipartition (i.e. $E_{\boldsymbol{u}}(k)=$ $\left.E_{\boldsymbol{b}}(k)\right)$ in the inertial range, which was predicted by Kraichnan [13] in the three-dimensional context and subsequently questioned by a number of authors [28-32], does not apply to the present case (see further detail below).

\section{B. Direct transfer of magnetic potential and equipartition of magnetic energy}

No appreciable inverse transfer of $\left\langle a^{2}\right\rangle$ has been observed across the series of simulations presented above. On the contrary, for the lower- $r_{0}$ cases, the redistribution of $\left\langle a^{2}\right\rangle$ from the 
reservoir (initially confined to $k \in[4,6]$ ) is in favor of the higher rather than the lower wave numbers. This direct transfer can be recognized from the spectrum of $\left\langle a^{2}\right\rangle$, which is given by $k^{-2} E_{\boldsymbol{b}}(k)$ and can be inferred from $E_{\boldsymbol{b}}(k)$ in Figs 6 and 7 . This spectrum is sufficiently steep and shallow for $k \lesssim 5$ and $k \gtrsim 5$, respectively, clearly indicating a biased distribution of $\left\langle a^{2}\right\rangle$ toward $k \gtrsim 5$. This is due to exceedingly strong magnetic stretching in these cases. A more quantitative knowledge of the direct transfer of $\left\langle a^{2}\right\rangle$ in the small $r_{0}$ limit can be gained from the results of the second and third set of simulations, the details of which were described earlier. For the remainder of this section, we present and discuss these results.

Fig. 8 shows the physical space representations of the vorticity and magnetic potential fields (at peak magnetic energy dissipation, $t=3$ ) for the system evolving from the spectrally localized vorticity field. Similarly, Fig. 9 shows these fields (at peak magnetic energy dissipation, $t=2.5$ ) for the system evolving from the fully developed vorticity field. These figures correspond to the case $r_{0}=1 / 256$. Because of limited scale resolution, the values of $|\omega|$ exceeding $3\left\langle\omega^{2}\right\rangle^{1 / 2}$ have been filtered out. As large vorticity is highly concentrated in space, this practice does not change the images in a recognizable manner. Clearly, the magnetic potential has evolved toward the small scales (see the initial potential field in Fig. 2 for a comparison). From the images of these fields for $r_{0}=1 / 1024$ and $r_{0}=1 / 4096$ (not shown), the tendency of $a$ to evolve toward the small scale becomes increasingly more pronounced for smaller $r_{0}$. A quantitative description of this behavior is given by Fig. 10, where the direct flux of $\left\langle a^{2}\right\rangle$, say $\Pi(k)$, is stronger for smaller $r_{0}$. It can be seen that $\Pi(k)$ has a slightly sharper peak for the case of developed velocity field. Note that for the case of localized initial velocity field and $r_{0}=1 / 4096, \Pi(k)$ is non-negative throughout, including the wave-number region lower than that of the initial magnetic reservoir. The reason is that in the early stage, a weak and brief inverse transfer had occurred and excited this region. The negative flux associated with this transfer is short-lived and, by the time $t=1$, is replaced by a non-negative flux.

Fig. 11 shows the magnetic and kinetic energy $E_{\boldsymbol{b}}(t)$ and $E_{\boldsymbol{u}}(t)$, as well as the dissipation rates $\epsilon_{\boldsymbol{b}}(t)$ and $\epsilon_{\boldsymbol{u}}(t)$ for $r_{0}=1 / 4096,1 / 1024$ and $1 / 256$ for the simulations evolving from a spectrally localized vorticity field. As $r_{0}$ decreases, $E_{\boldsymbol{b}}(t), \epsilon_{\boldsymbol{b}}(t)$ and $\epsilon_{\boldsymbol{u}}(t)$ grow more rapidly, achieving a higher peak in a shorter time, Intuitively, this time has some bearing on the turnover time of the turbulence. Unfortunately, a quantitative knowledge of this issue is not possible due to insufficient data. 

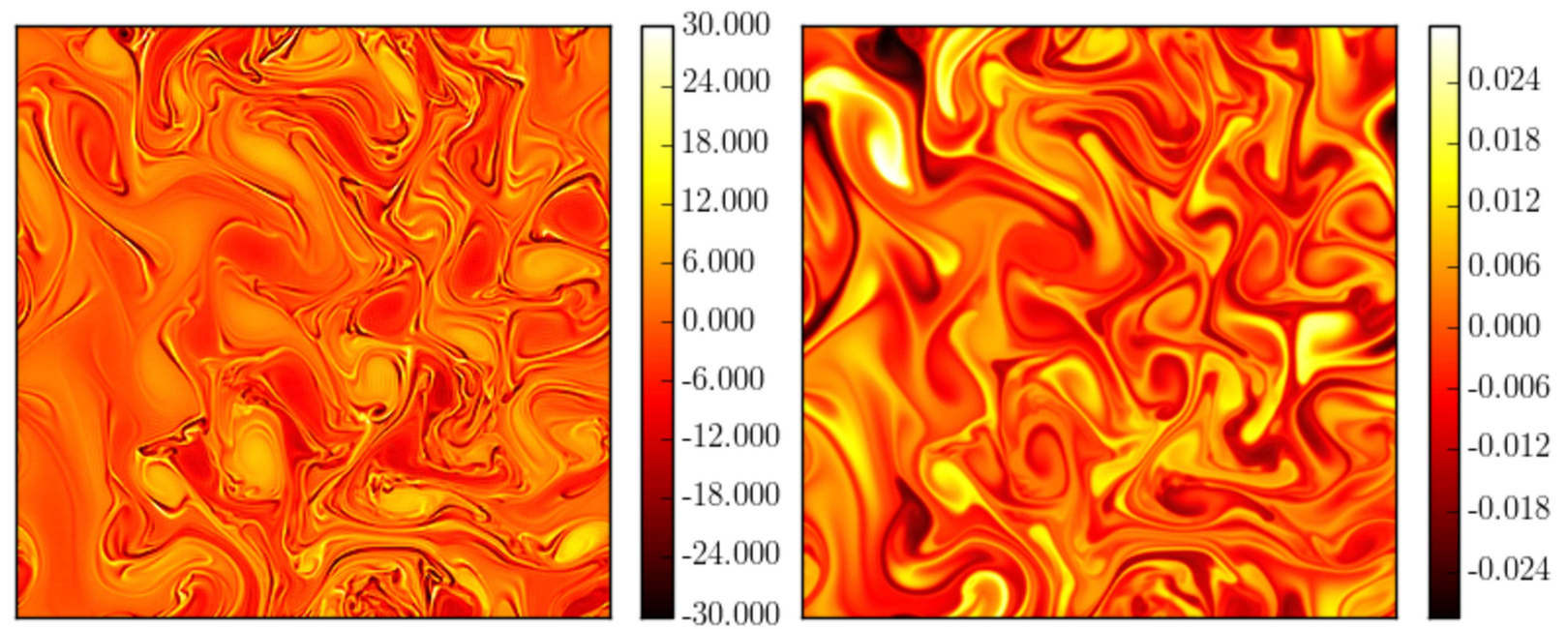

FIG. 8: (Color online) Vorticity (left) and magnetic potential (right) fields at peak magnetic energy dissipation, $t=3$, for $r_{0}=1 / 256$ for simulations evolving from a vorticity field spectrally confined within the wave-number interval $[5,7]$. The vorticity field is plotted with values between $-3\left\langle\omega^{2}\right\rangle^{1 / 2}$ and $3\left\langle\omega^{2}\right\rangle^{1 / 2}=30$, while the maximum value is $\approx 140$.

Fig. 12 compares the magnetic energy, kinetic energy, magnetic energy dissipation and kinetic energy dissipation for the two different initial conditions for the case $r_{0}=1 / 4096$. As mentioned above the initial fully developed vorticity field has less enstrophy, meaning that the kinetic energy dissipation rate is lower throughout.

Fig. 13 shows the magnetic and kinetic energy spectra at the time of maximum magnetic energy dissipation. Across the board, the spectrum $E_{\boldsymbol{b}}(k)$ has a positive slope and becomes wider for smaller $r_{0}$. Remarkably, this positive slope appears to tend to unity in the small- $r_{0}$ limit. Such limiting scaling corresponds to equipartition of magnetic energy among individual Fourier modes and equipartition of magnetic potential variance among the wave-number octaves of the inertial range, respectively. The spectrum $E_{\boldsymbol{u}}(k)$ possesses two distinct ranges. At lower wave numbers $E_{\boldsymbol{u}}(k)$ is significantly steeper, which is slightly more distinct in the case having evolved from a fully developed vorticity field, then shallows to approximate the classical Kolmogorov $k^{-5 / 3}$ spectrum. Evidently, the overall spectrum is poorly represented by a single scaling exponent. Note that the difference in the initial velocity field has little effect on $E_{\boldsymbol{u}}(k)$ and $E_{\boldsymbol{b}}(k)$. Fig. 14 replots the magnetic and kinetic 

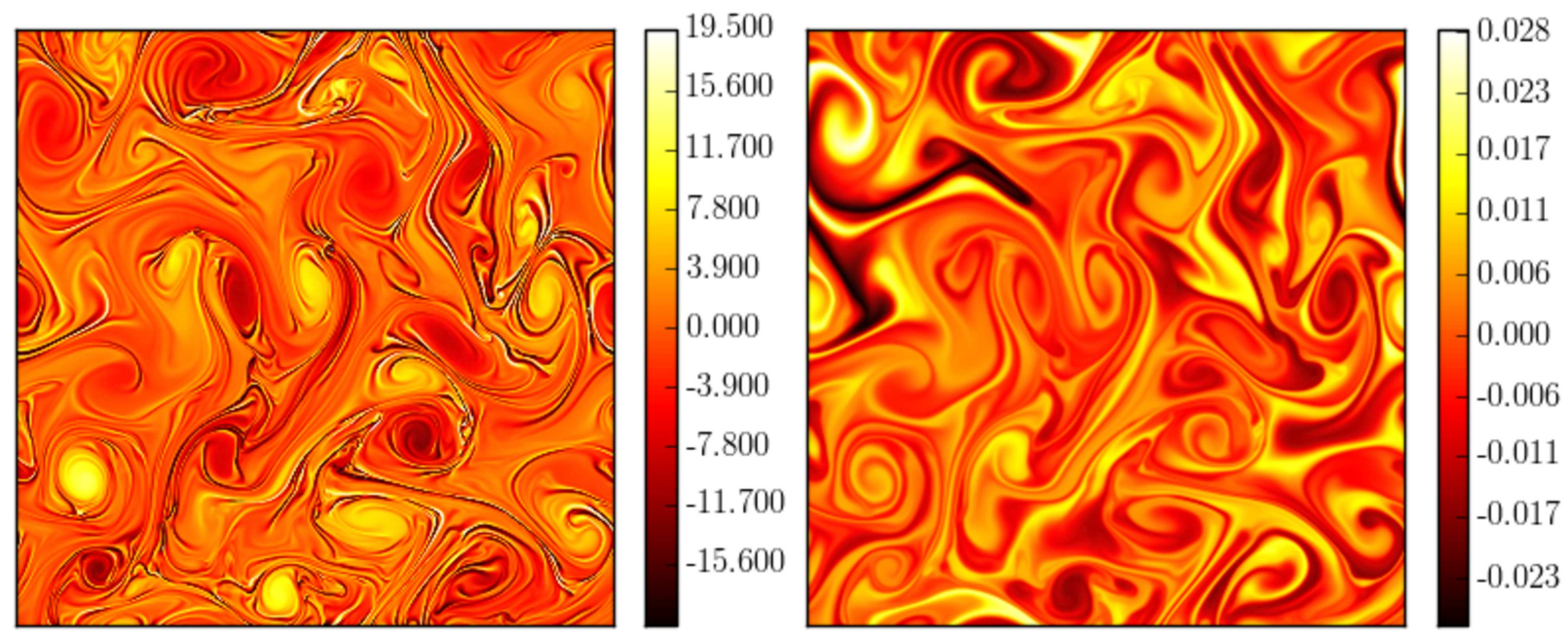

FIG. 9: (Color online) Vorticity (left) and magnetic potential (right) fields at peak magnetic energy dissipation, $t=2.5$, for $r_{0}=1 / 256$ for simulations evolving from a fully developed vorticity field. The vorticity field is plotted with values between $-3\left\langle\omega^{2}\right\rangle^{1 / 2}$ and

$$
3\left\langle\omega^{2}\right\rangle^{1 / 2}=19.5 \text {, while the maximum value is } \approx 87 .
$$

energy spectra for the case $r=1 / 4096$ from Fig. 13 for comparison.

Some remarks about the asymptotic behavior of $E_{\boldsymbol{b}}(k)$ are in order. Compelling evidence from Fig. 13 suggests the spectrum $E_{\boldsymbol{b}}(k)=C k^{1}$, which extends to an ever-higher wave number, say $k_{m}$, as $r_{0} \rightarrow 0$ (and in the limit of ideal dynamics). For such a spectrum, the conservation of $\left\langle a^{2}\right\rangle$ requires

$$
\left\langle a_{0}^{2}\right\rangle=C \int_{k_{0}}^{k_{m}} k^{-1} d k=C \ln \left(\frac{k_{m}}{k_{0}}\right),
$$

where $k_{0}$ can be taken as the system's smallest wave number. The divergence of $k_{m}$ implies a logarithmic decrease of $C$, i.e. a logarithmic collapse of $E_{\boldsymbol{b}}(k)$. Meanwhile the magnetic energy is given by

$$
E_{\boldsymbol{b}}=C \int_{k_{0}}^{k_{m}} k^{1} d k \approx C k_{m}^{2}=\frac{\left\langle a_{0}^{2}\right\rangle}{\ln \left(k_{m} / k_{0}\right)} k_{m}^{2}
$$

Apparently, this corresponds to no upper bound for $E_{\boldsymbol{b}}$ in the limit $r_{0} \rightarrow 0$, or equivalently, the kinetic energy available for conversion is unlimited. Note that this theoretical picture would become complete if a dependence of $k_{m}$ on $r_{0}$ could be established. 

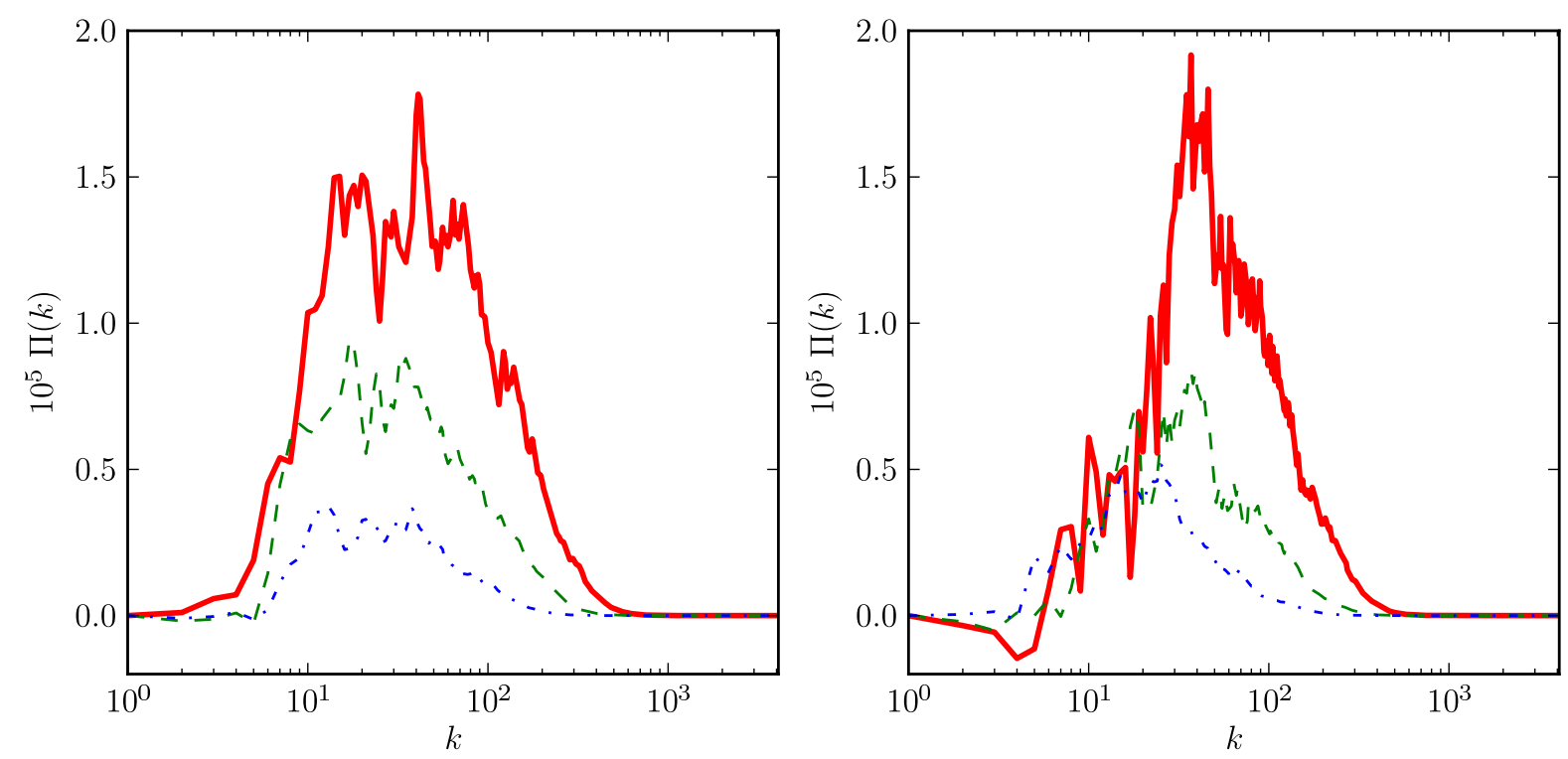

FIG. 10: (Color online) Flux of magnetic potential variance at the time of peak magnetic energy dissipation for $r_{0}=1 / 256$ (dashed-dotted), $r_{0}=1 / 1024$ (dashed) and $r_{0}=1 / 4096$ (solid). These occur at $t=3, t=1.6$ and $t=1$ respectively for evolution from a localized initial vorticity field (left), and $t=2.4, t=1.8$ and $t=1.1$ for evolution from a fully developed vorticity field (right).

The present spectrum $E_{\boldsymbol{b}}(k)=C k$ may be considered as the analog of the Kazantsev $[7,33]$ spectrum $k^{3 / 2}$ in three dimensions. However, in the latter case accumulation of magnetic energy in the inertial range is of a lesser extent, quite short of equipartition among the Fourier modes. One may attribute this to relatively strong direct energy transfer in three dimensions.

Cho et al. [34] simulated small-scale dynamo in three-dimensional magnetohydrodynamic turbulence driven by large-scale mechanical forcing. In the weak field regime, these authors found that $E_{\boldsymbol{b}}(k)$ scaled in a similar manner to the Kazantsev spectrum (see their Fig. 4). Hence equipartition of magnetic energy among the Fourier modes in the inertial range found presently may not apply to the three-dimensional case.

As observed by Cho et al. [34], dynamo primarily occurs near the truncation wave number during the early stage. In our simulations, energy conversion initially occurs in the vicinity of the large-scale magnetic reservoir and subsequently proceeds to smaller scales. This is the case for both spectrally confined and fully developed initial velocity fields. Fig. 15 

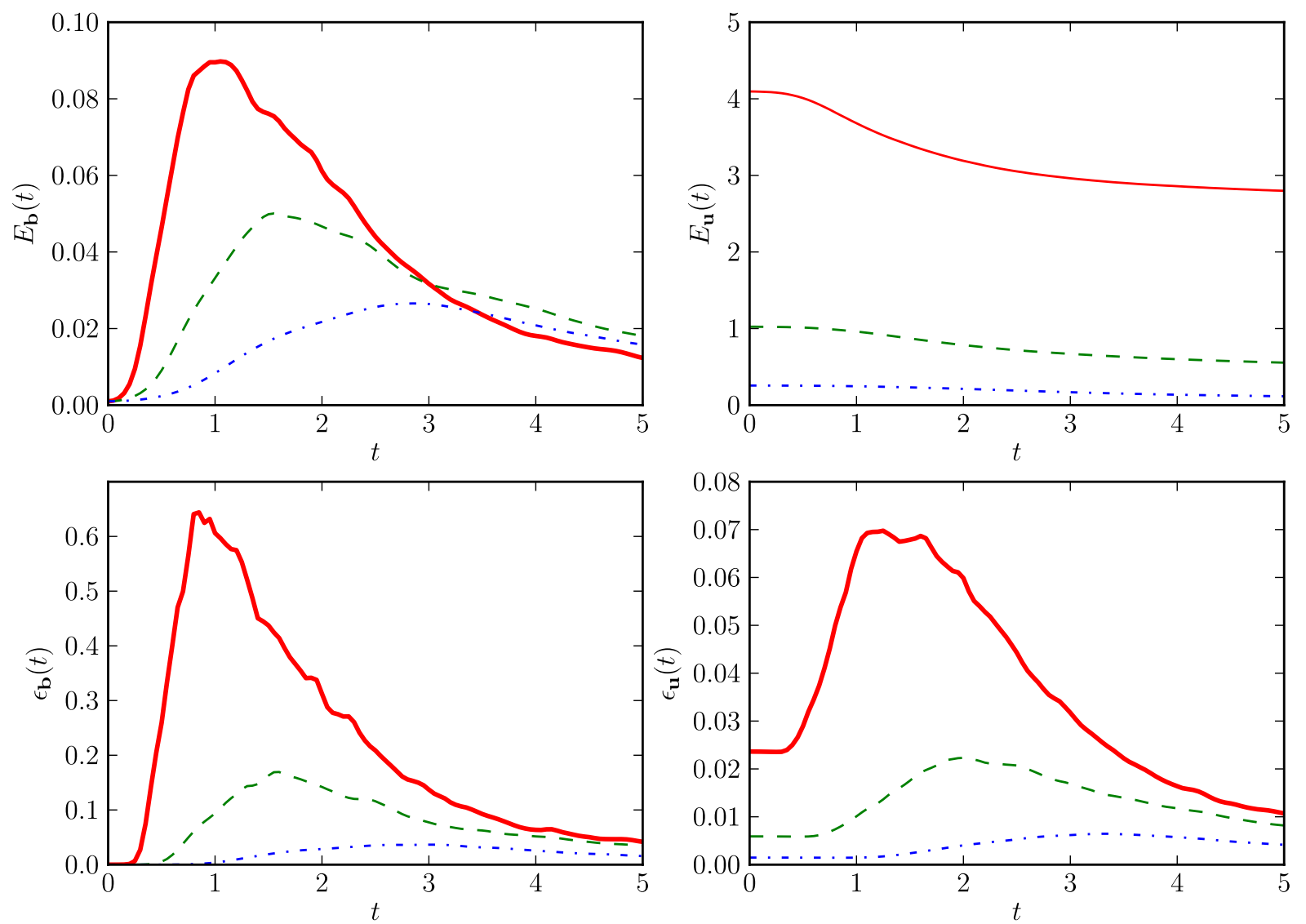

FIG. 11: (Color online) Magnetic energy $E_{\boldsymbol{b}}$, kinetic energy $E_{\boldsymbol{u}}$, magnetic energy dissipation rate $\epsilon_{\boldsymbol{b}}$ and kinetic energy dissipation rate $\epsilon_{\boldsymbol{u}}$ for $r_{0}=1 / 256$ (dashed-dotted), 1/1024 (dashed) and 1/4096 (solid) for spectrally localized initial conditions.

quantitavely describes this behaviour by showing $E_{\boldsymbol{b}}(k)$ at three different times during the evolution.

The residual energy $E_{\boldsymbol{b}}(k)-E_{\boldsymbol{u}}(k)$ in the inertial and dissipation ranges is positive (see Fig. 16). The wave number at which this energy becomes positive increases as $r_{0}$ is decreased. In the picture described by the preceding paragraph, this wave number grows (presumably logarithmically) without bound in the limit $r_{0} \rightarrow 0$.

\section{CONCLUDING REMARKS}

We have investigated dynamical aspects and inertial-range scaling laws of two-dimensional magnetohydrodynamic turbulence in the weak field regime, extending a previous study 

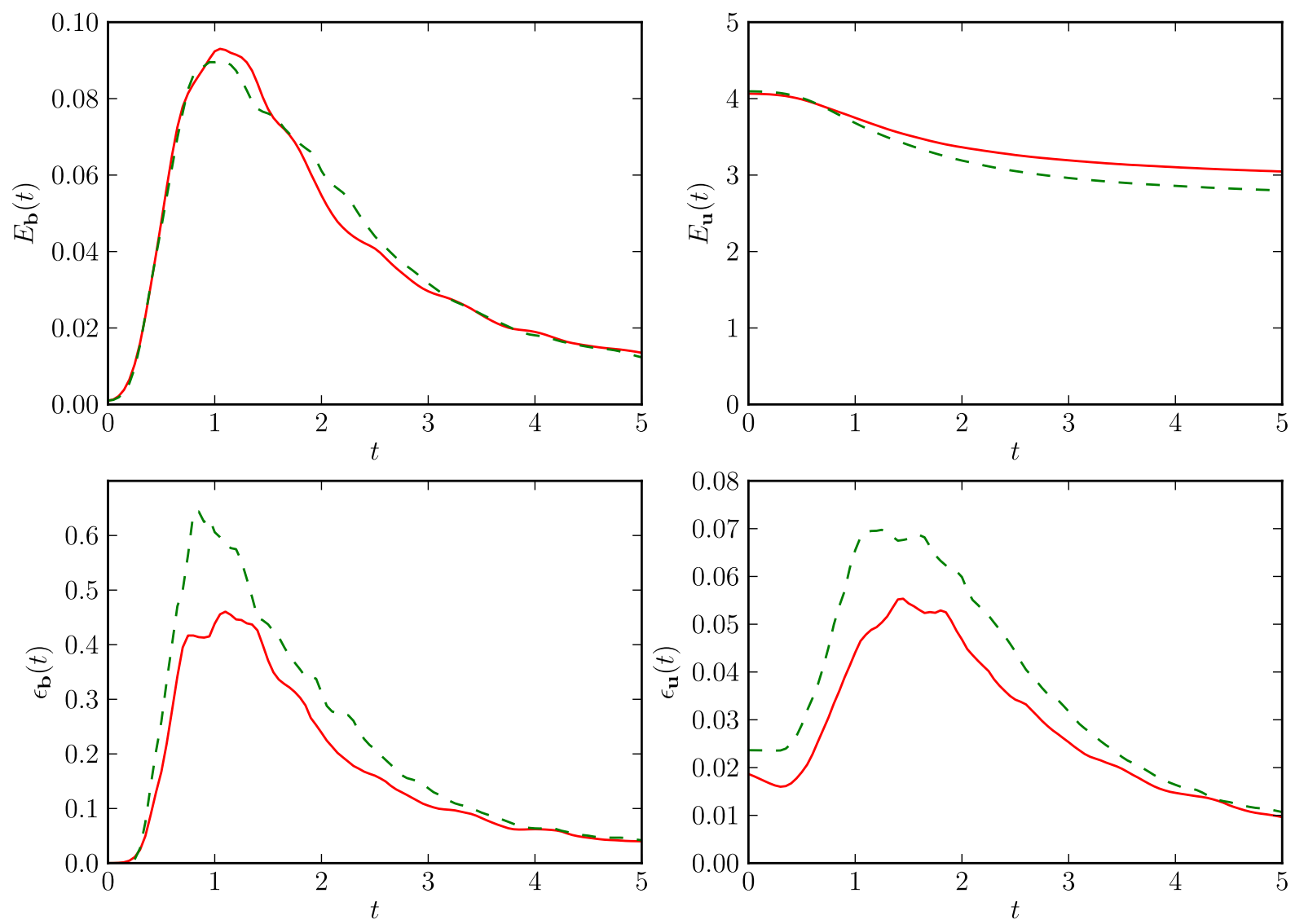

FIG. 12: (Color online) Magnetic energy $E_{\boldsymbol{b}}$, kinetic energy $E_{\boldsymbol{u}}$, magnetic energy dissipation rate $\epsilon_{\boldsymbol{b}}$ and kinetic energy dissipation rate $\epsilon_{\boldsymbol{u}}$ for $r_{0}=1 / 4096$ for a fully developed (solid) and spectrally localized (dashed) initial velocity fields.

by Blackbourn and Tran [17] in several directions. This has been achieved by doubling the numerical resolution and broadening the initial magnetic-to-kinetic energy ratio $r_{0}$ by almost two more decades toward the small $r_{0}$ limit, i.e. toward the weak field regime. The dynamics of this regime is characterized by strong magnetic stretching, with intense conversion of kinetic to magnetic energy. The present focus is on quantitative effects of $r_{0}$ on the inertial-range dynamics and scaling laws. Three series of direct numerical simulations up to $8192 \times 8192$ grid points at unity magnetic Prandtl number and over $r_{0}=1 / 4,1 / 16,1 / 64,1 / 256,1 / 1024,1 / 4096$ were carried out. In one of these series, the initial total energy was fixed at unity, while in the other two the initial magnetic energy was fixed at 0.001 . For the latter case two different forms of the initial velocity field were 

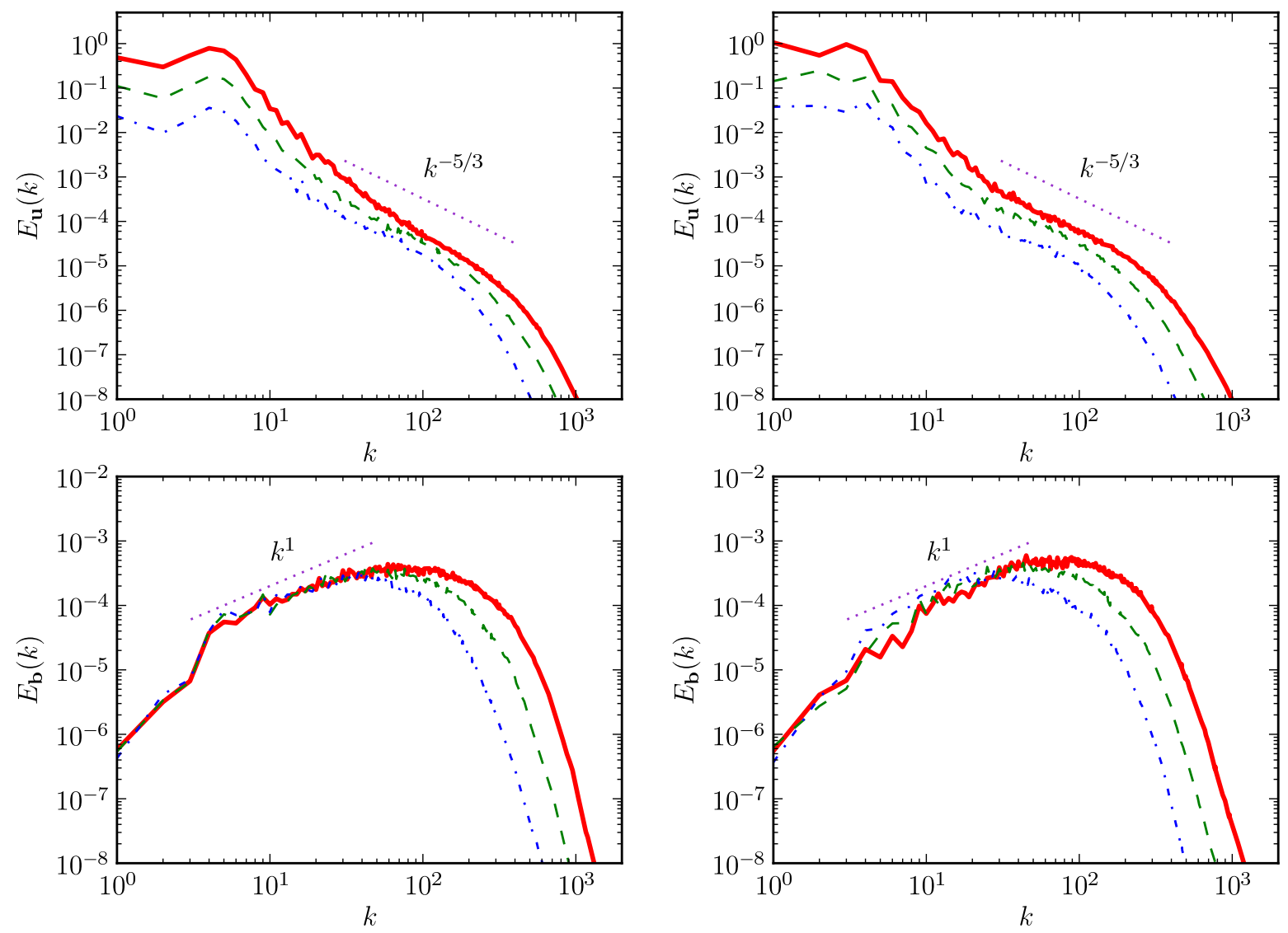

FIG. 13: (Color online) Kinetic energy spectrum $E_{\boldsymbol{u}}(k)$ and magnetic energy spectrum $E_{\boldsymbol{b}}(k)$ at peak magnetic energy dissipation for $r_{0}=1 / 256$ (dashed-dotted), $r_{0}=1 / 1024$ (dashed) and $r_{0}=1 / 4096$ (solid) for spectrally confined (left) and fully developed (right) initial velocity fields.

used, one of which was spectrally localized while the other was fully developed. This setup allows for probing into detailed effects of the spectral distribution of the velocity field and its increasing strength on a fixed magnetic seed.

The total energy spectrum $E(k)$ has been observed to be much shallower than previously thought. This spectrum depends on $r_{0}$ since its constituents, i.e. the magnetic and kinetic energy spectra $E_{\boldsymbol{b}}(k)$ and $E_{\boldsymbol{u}}(k)$, each strongly depends on $r_{0}$. In particular, $E_{\boldsymbol{b}}(k)$ becomes shallower as $r_{0}$ is decreased. The extent of this shallow spectrum is limited and becomes broader for smaller $r_{0}$, probably without bound in the limit $r_{0} \rightarrow 0$ (with fixed initial magnetic energy). Furthermore, in this limit, the slope of $E_{\boldsymbol{b}}(k)$ appears to tend to +1 . This 

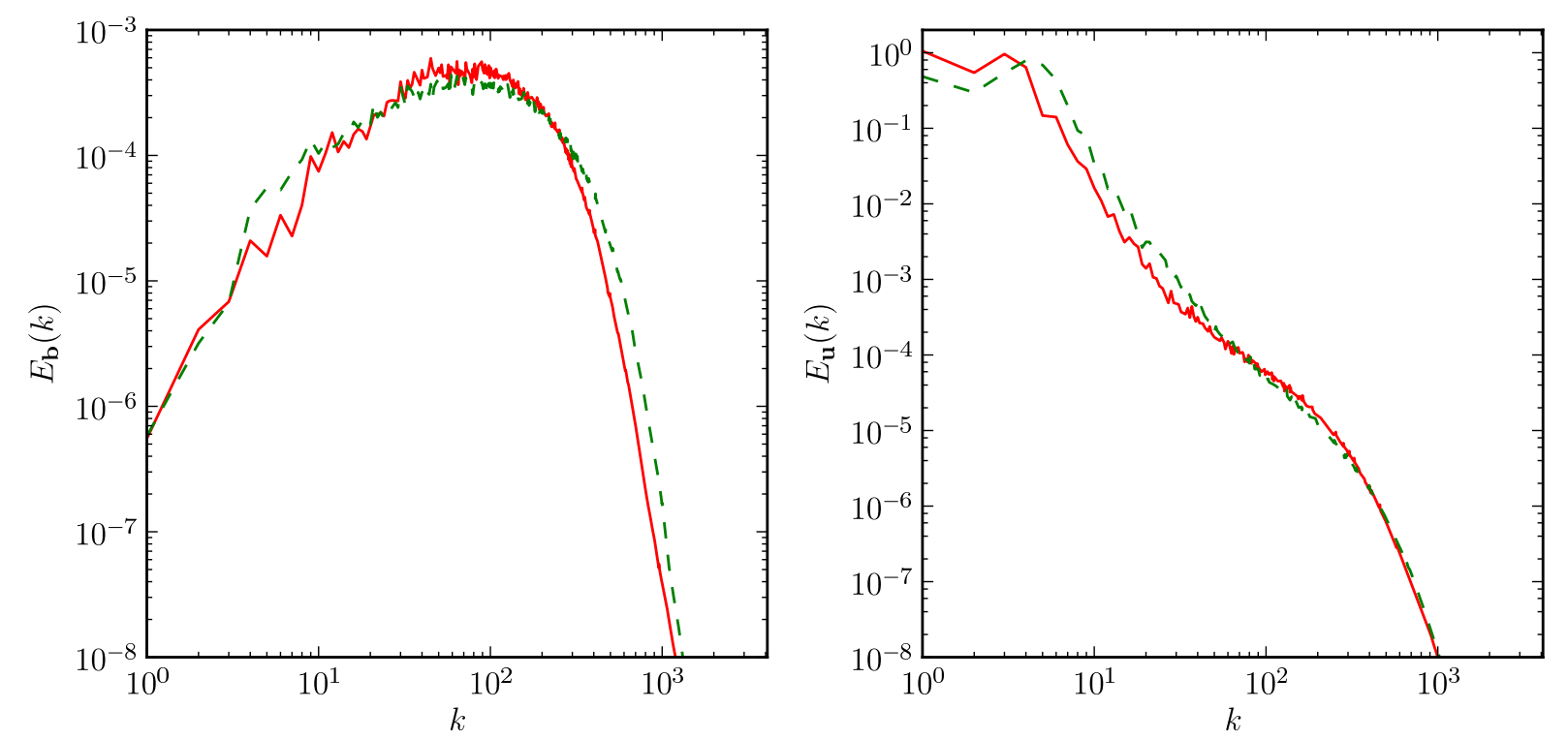

FIG. 14: (Color online) Magnetic (left) and kinetic (right) energy spectra for $r_{0}=1 / 4096$ at the time of maximum magnetic energy dissipation for turbulence evolving from spectrally confined (dashed) and fully developed (solid) initial velocity fields.

corresponds to equipartition of magnetic energy among the Fourier modes, or equivalently, equipartition of magnetic potential variance among the wave-number octaves of the inertial range. The latter spectral distribution is reminiscent of that of the variance of a passive scalar advected by large-scale flows. Note, however, that the observed dynamical resemblance between a passive scalar and the magnetic potential is rather superficial as there are fundamental differences. One is that antidynamo interaction, which is an integral part of the dynamics of the magnetic potential, qualifies it as an active scalar in its own right. Another difference is that advection can amplify the mean-square gradient of a passive scalar without bound in the large-time limit, whereas $E_{\boldsymbol{b}}(t)$ is uniformly bounded in time. This means that the direct transfer of magnetic potential, which can be said to be induced by strong magnetic stretching, is relatively less spontaneous than that of its passive counterpart.

Kinetic-to-magnetic energy conversion, a manifestation of magnetic stretching, may be considered the primary interaction, while the reverse process is of a secondary nature. Hence, it is not surprising that the inertial and dissipation ranges have been found to be more strongly excited magnetically than mechanically.

Some arguments in the present study may apply to three-dimensional magnetohydrody- 

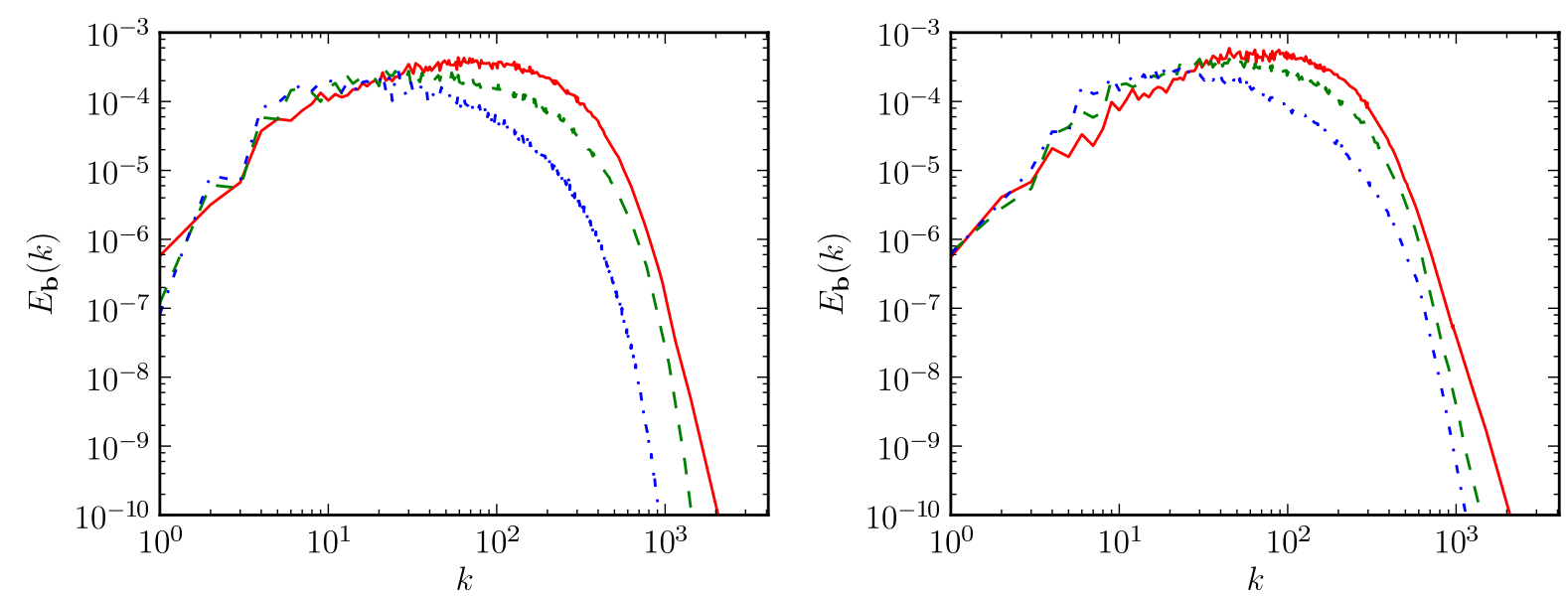

FIG. 15: (Color online) Magnetic energy spectra for $r_{0}=1 / 4096$ at one-third the time of maximum dissipation (dashed-dotted), half the time of maximum dissipation (dashed) and the time of maximum dissipation (solid) for turbulence evolving from spectrally confined (left) and fully developed (right) initial velocity fields.

namic turbulence. Intuitively, dynamo action in three dimensions and kinetic-to-magnetic energy conversion in two dimensions can be expected to bring about similar effects. These include the deposition of the converted magnetic energy in the inertial range and the weakening of the velocity field. Note, however, that in three dimensions, dynamo action is not known to be constrained by the conservation of a positive-definite quadratic quantity other than the total energy. An implication is that if the magnetic energy grows without bound in the limit $r_{0} \rightarrow 0$ (with fixed initial magnetic energy), its spectrum need not collapse as in the present case.

\section{ACKNOWLEDGEMENTS}

The work reported here was presented at ETC13 in Lyon and represents part of L.A.K.B.'s Ph.D. thesis, which was supported by an EPSRC postgraduate studentship. L.A.K.B. was further supported by an EPSRC doctoral prize. The authors would like to thank Rodion Stepanov for suggesting a comprehensive comparison between the cases of spectrally localized 

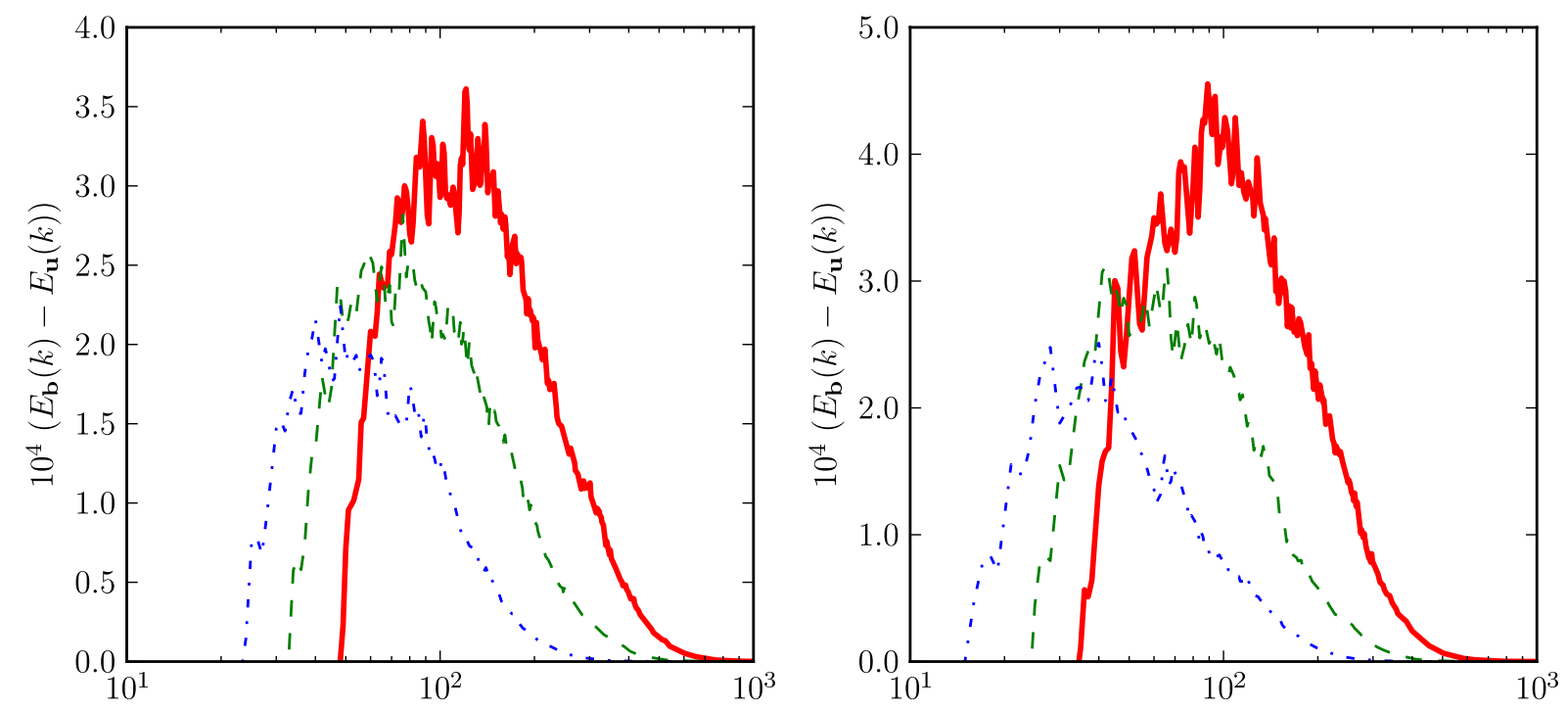

FIG. 16: (Color online) Residual energy $E_{\boldsymbol{b}}(k)-E_{\boldsymbol{u}}(k)$ versus $k$ at peak magnetic energy dissipation for $r_{0}=1 / 256$ (dashed-dotted), $r_{0}=1 / 1024$ (dashed) and $r_{0}=1 / 4096$ (solid), for spectrally confined (left) and fully developed (right) initial velocity fields.

and fully developed initial velocity fields.

[1] A. Brandenburg, Astrophys. J. 741, 92 (2011).

[2] A. Brandenburg, Astron. Nachr. 332, 51 (2011).

[3] F. Cattaneo and S. M. Tobias, J. Fluid Mech. 621, 205 (2009).

[4] F. Cattaneo and S. I. Vainshtein, Astrophys. J. 376, L21 (1992).

[5] A. V. Gruzinov and P. H. Diamond, Phys. Rev. Lett. 72, 1651 (1994).

[6] N. E. L. Haugen, A. Brandenburg, and W. Dobler, Rhys. Rev. E 70, 016308 (2004).

[7] A. P. Kazantsev, Sov. Phys. JETP 26, 1031 (1968).

[8] R. M. Kulsrud and S. W. Anderson, Astrophys. J. 396, 606 (1992).

[9] P. D. Mininni, Phys. Rev. E 76, 026316 (2007).

[10] A. A. Schekochihin, S. C. Cowley, S. F. Taylor, J. L. Maron, and J. C. McWilliam, Astrophys. J. 612, 276 (2004).

[11] S. M. Tobias and F. Cattaneo, J. Fluid Mech. 601, 101 (2008). 
[12] P. S. Iroshnikov, Sov. Astron. 7, 566 (1964).

[13] R. H. Kraichnan, Phys. Fluids 8, 1385 (1965).

[14] S. Sridhar and P. Goldreich, Astrophys. J. 432, 612 (1994).

[15] P. Goldreich and S. Sridhar, Astrophys. J. 438, 763 (1995).

[16] C. V. Tran, X. Yu, and L. A. K. Blackbourn, J. Fluid Mech. 725, 195 (2013).

[17] L. A. K. Blackbourn and C. V. Tran, J. Fluid Mech. 703, 238 (2012).

[18] S. Galtier, S. V. Nazarenko, A. C. Newell, and A. Pouquet, J. Plasma Phys. 63, 447 (2000).

[19] D. Fyfe and D. Montgomery, J. Plasma Phys. 16, 181 (1976).

[20] A. Pouquet, J. Fluid Mech. 88, 1 (1978).

[21] D. Biskamp and U. Bremer, Phys. Rev. Lett 72, 3819 (1993).

[22] Z. Lei and Y. Zhou, Discrete Contin. Dyn. Syst. 25, 575 (2009).

[23] C. Cao and J. Wu, Adv. Math. 226, 1803 (2011).

[24] C. V. Tran, Phys. Rev. E 78, 036310 (2008).

[25] T. Y. Hou and R. Li, J. Comput. Phys. 226, 379 (2007).

[26] C. V. Tran and X. Yu, Phys. Rev. E 85, 066323 (2012).

[27] D. Biskamp and H. Welter, Phys. Fluids B 1, 1964 (1989).

[28] R. Grappin, A. Pouquet, and J. Léorat, Astron. Astrophys. 126, 51 (1983).

[29] W. Müller and R. Grappin, Phys. Rev. Lett. 95, 114502 (2005).

[30] J. J. Podesta, D. A. Roberts, and M. L. Goldstein, Astrophys. J. 664, 543 (2007).

[31] S. Boldyrev and J. C. Perez, Phys. Rev. Lett. 103, 225010 (2009).

[32] J. A. Tessein, C. W. Smith, B. T. MacBride, W. H. Matthaeus, M. A. Forman, and J. E. Borovsky, Astrophys. J. 692, 684 (2009).

[33] A. Brandenburg and K. Subramanian, Phys. Rep. 417, 1 (2005).

[34] J. Cho, E. T. Vishniac, A. Beresnyak, A. Lazarian, and D. Ryu, Astrophys. J. 693, 1449 (2009). 